\title{
Correlation between Claudin-18 expression and clinicopathological features and prognosis in patients with gastric cancer
}

\author{
Yufeng Lu", Tielong Wü, Yingyue Sheng, Yuanyuan Dai, Beilei Xia, Yuzheng Xue \\ Department of Gastroenterology, Affiliated Hospital of Jiangnan University, Wuxi, China \\ Contributions: (I) Conception and design: Y Lu, T Wu; (II) Administrative support: Y Xue; (III) Provision of study materials or patients: Y Lu, Y Dai; \\ (IV) Collection and assembly of data: Y Sheng; (V) Data analysis and interpretation: B Xia; (VI) Manuscript writing: All authors; (VII) Final approval \\ of manuscript: All authors. \\ \#These authors contributed equally to the work. \\ Correspondence to: Yuzheng Xue. Department of Gastroenterology, Affiliated Hospital of Jiangnan University, Wuxi 214041, China. \\ Email: xueyz001@163.com.
}

Background: To investigate the correlation between Claudin-18 expression and the clinicopathological features and prognosis of gastric cancer.

Methods: A total of 63 patients who underwent gastric cancer surgery from December 2012 to June 2013 were recruited as the research participants. The clinicopathological data and prognostic information of the participants were collected, and expression levels of Claudin-18 in gastric cancer and adjacent tissues were detected by immunohistochemical (IHC) staining. The correlation between Claudin-18 expression and clinicopathological features of gastric cancer patients was analyzed. The Cox regression model was used to analyze the risk factors associated with the prognosis of gastric cancer patients.

Results: The expression of Claudin-18 was positive in 35 (55.6\%) of the participants' gastric cancer tissues, which was significantly lower than that in the adjacent tissues (51 tissues/81.0\%), and the difference was statistically significant $(\mathrm{P}=0.002)$. In addition, the IHC staining score of Claudin-18 expression in gastric cancer tissues $(1.49 \pm 1.69)$, was significantly lower than that in the adjacent tissues $(4.61 \pm 1.81)$, and the difference was statistically significant $(\mathrm{P}=0.016)$, The incidence of nerve invasion in patients with low expression of Claudin-18 was significantly higher than that in patients with high expression of Claudin-18 $(\mathrm{P}=0.038)$. In addition, univariate Cox regression analysis showed that nerve invasion, Claudin-18 staining score, tumor size, and positive count of lymph nodes were risk factors associated with the survival of gastric cancer patients. Multivariate Cox regression analysis showed that Claudin-18 staining score and tumor size were independent risk factors associated with the survival of gastric cancer patients. The overall survival (OS) of patients with low Claudin-18 staining score or larger tumor size was significantly reduced.

Conclusions: The low expression of Claudin-18 was closely related to nerve invasion in gastric cancer, which indicated the poor clinical prognosis of gastric cancer patients.

Keywords: Claudin-18; gastric cancer; nerve invasion; prognosis

Submitted Sep 14, 2020. Accepted for publication Dec 16, 2020.

doi: 10.21037/jgo-20-463

View this article at: http://dx.doi.org/10.21037/jgo-20-463

(C) Journal of Gastrointestinal Oncology. All rights reserved. 


\section{Introduction}

Gastric cancer is one of the most common malignant tumors, and is a threat to human life and health. The incidence and mortality of gastric cancer in China are higher than those in western countries (1). In 2017, the National Cancer Center released a report on the current situation of gastric cancer in China (1). In 2012, there were 951,000 new cases of gastric cancer and 723,000 deaths. Among them, Chinese accounted for nearly half of the new cases and deaths worldwide.

Surgery is the most effective treatment for early gastric cancer. For patients with advanced gastric cancer, especially those who have missed their chance for radical cure, mainly rely on chemotherapy, radiotherapy, and neoadjuvant chemoradiotherapy, among others. However, these treatments are nonspecific, with limited clinical efficacy and serious adverse events. Further, the long-term survival and prognosis of patients are not significantly improved as a result of such treatments (2). Targeted therapy is a hot spot in tumor combination therapy, which can not only improve clinical efficacy, but also minimize side effects. At present, several targeted therapeutic drugs have been approved for clinical use, and have been successfully used in the treatment of melanoma and non-small cell lung cancer (3). However, for gastric cancer, there is still a lack of effective therapeutic targets (4), and tumor recurrence and metastasis are still the main factors affecting the clinical prognoses of advanced gastric cancer patients (5).

Tight junction is a junction complex between epithelial and endothelial cells, which forms a material barrier around the cells, and plays an important role in cell polarity, permeability, adhesion, and regulation of cell proliferation and differentiation (6). The Claudins protein family is a human tight junction protein, whose function is to maintain the stability of epithelial cells (7). Studies have shown that Claudins are often down-regulated in cancer tissues, resulting in abnormal tight junction of cells, and decreased cell differentiation and polarity, which is closely related to the occurrence and development of tumors (8). Claudin-18 protein is a member of the Claudins protein family. A previous study showed that a monoclonal antibody against Claudin-18 combined with EOX (epirubicin + oxaliplatin + capecitabine) is feasible and safe in the treatment of patients with advanced or metastatic gastroesophageal cancer (9). However, the role of Claudin-18 in the development of gastric cancer is still unknown.

The current study aimed to investigate the expression and distribution of Claudin-18 in gastric cancer, and analyze its correlation with clinicopathological features and prognosis of gastric cancer. We aimed to gather knowledge to improve the screening of potential molecular therapeutic targets for gastric cancer, and provide a theoretical basis for the development of individualized therapy of gastric cancer and the development of small molecule targeted drugs, so as to further improve the clinical efficacy and prognosis for patients with gastric cancer.

We present the following article in accordance with the REMARK reporting checklist (available at http://dx.doi. org/10.21037/jgo-20-463).

\section{Methods}

\section{Participant enrollment}

A total of 63 patients with gastric cancer, who were treated at our hospital from December 2012 to June 2013, were recruited as the research participants. Enrollment criteria included pathological confirmation of the specimens and no radiotherapy or chemotherapy before operation. The clinical stage was defined in accordance with the $7^{\text {th }}$ edition of clinical staging standards.

All procedures performed in this study involving human participants were in accordance with the Declaration of Helsinki (as revised in 2013). The study was approved by the ethics committee of our hospital before implementation (No. IEC20200142). The participants gave their written informed consent before participation in the study.

\section{Data collection}

Clinical and pathological data (age, gender, operation time, tumor size, clinical stage, vascular invasion, nerve invasion, total count of lymph nodes, and positive count of lymph nodes) were collected.

\section{Follow up}

The survival situation of patients was obtained by telephone interview.

\section{Immunobistochemical (IHC) staining}

The tissue wax was sliced by a slicer to a thickness of $5 \mu \mathrm{m}$. The flattened wax was fixed on the glass slide with adhesive to make paraffin sections. The paraffin slices were 
Table 1 Clinicopathological information of the patients

\begin{tabular}{lc}
\hline Clinicopathological information & Value \\
\hline Gender (n) & 49 \\
Male & 14 \\
Female & $65.94 \pm 11.44$ \\
Age (years) & \\
Vascular invasion (n) & 13 \\
Positive & 50 \\
Negative & \\
Nerve invasion (n) & 9 \\
Positive & 54 \\
Negative & \\
Clinical stage (n) & 9 \\
I & $15.94 \pm 9.18$ \\
II & $6.11 \pm 7.26$ \\
III & \\
IV & 36 \\
Tumor size (cm) & 17 \\
Total count of lymph nodes (count) & \\
Positive count of lymph nodes (count) & \\
\hline
\end{tabular}

roasted for $90 \mathrm{~min}\left(70{ }^{\circ} \mathrm{C}\right)$, and dewaxing was conducted twice in xylene, 10 min each time; $100 \%, 95 \%$, and $85 \%$ alcohol were successively passed through the cylinder for 5 min each time; the slides were washed with natural water until the they were clear. In order to block endogenous peroxidase, slides were immersed in $3 \%$ hydrogen peroxide for $8 \mathrm{~min}$; washed 3 times in distilled water, added to a temporary citrate buffer solution, heated at $95^{\circ} \mathrm{C}$ for $15 \mathrm{~min}$ in a microwave oven, and cooled at room temperature. The primary antibody of Claudin-18 (1:500, Abcam, Cambridge, MA, USA) was incubated in a wet box at $4{ }^{\circ} \mathrm{C}$ overnight, then removed from the humidification box and left at room temperature for 10-20 mins. It was then washed 3 times in phosphate buffer saline (PBS); the secondary antibody (1:10,000, Jackson ImmunoResearch Laboratories Inc., West Grove, PA, USA) was added and incubated in an oven at a constant temperature of $37^{\circ} \mathrm{C}$ for $30 \mathrm{~min}$; followed by washing 3 times in PBS buffer solution, and then diaminobenzidine (DAB) was used for color development. It was then dyed with hematoxylin for $3 \mathrm{~s}$, differentiated in lithium carbonate for $2 \mathrm{~s}$; and finally, dehydrated with gradient ethanol, xylene was used for transparency, and it was then sealed with neutral gum.

\section{IHC staining score}

We took 3 visual fields from each slide and calculated the average. The staining score was averaged according to the dyeing area and degree of dyeing; the dyeing area: 0.0 $5.0 \%=0$ points, $5.1-25.0 \%=1$ point, $25.1-50.0 \%=2$ points, $50.1-75.0 \%=3$ points, and $\geq 75.0 \%=4$ points. The dyeing intensity was judged according to the color depth; no staining $=0$ points, light yellow $=1$ point, brown yellow $=2$ points, brown marks $=3$ points. The final total score was calculated according to the positive staining area $\times$ positive staining intensity.

\section{Statistical analysis}

The SPSS software version 18.0 (IBM Corp., Armonk, NY, USA) was used for statistical analysis. The measurement data were expressed as mean \pm standard deviation, and the difference between the 2 groups was analyzed by $t$-test. The count data were expressed as $\mathrm{n} / \%$, and the difference between the 2 groups was analyzed by chi-square $\left(\chi^{2}\right)$ test. The Cox regression model was used to screen the risk factors associated with the prognosis of gastric cancer patients with. A difference of $\mathrm{P}<0.05$ was considered as statistically significant.

\section{Results}

\section{Clinicopathological information of the patients}

All the patients are diagnosed with gastric adenocarcinoma. The clinicopathological information of the 63 participants is listed in Table 1.

\section{Expression of Claudin-18 in gastric cancer}

The expression of the Claudin-18 protein in gastric cancer and adjacent tissues of 63 patients was detected by IHC. The results showed that Claudin-18 was positively expressed in $35(55.6 \%)$ gastric cancer tissues and $51(81.0 \%)$ adjacent tissues. The positive expression rate of Claudin-18 in gastric cancer tissues was significantly lower than that in adjacent tissues $\left(\chi^{2}=9.377, \mathrm{P}=0.002\right)$. In addition, the IHC staining score of Claudin-18 in gastric cancer tissues was $(1.49 \pm 1.69)$, which was significantly lower than that in adjacent tissues 


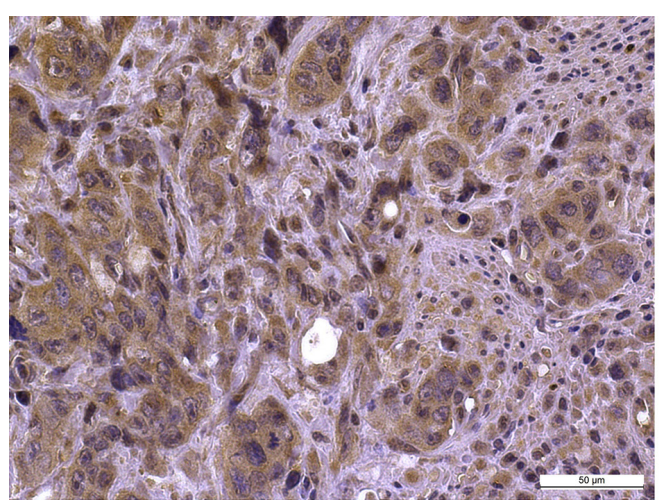

Gastric cancer tissue

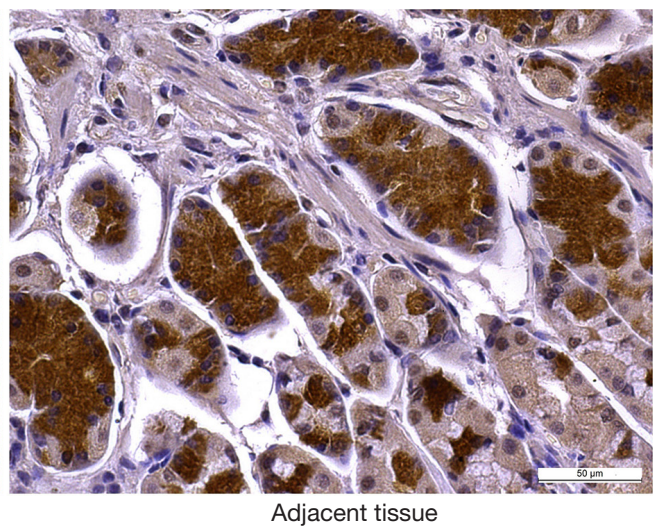

Figure 1 Claudin-18 expression in gastric cancer and adjacent tissues by immunohistochemical staining (Diaminobenzidine staining).

$(4.61 \pm 1.81)$, and the difference was statistically significant $(t=6.101, \mathrm{P}=0.016)$ (Figure 1).

\section{Correlation between Claudin-18 expression and clinicopathological features of gastric cancer}

According to the expression level of Claudin-18 in gastric cancer tissues, the patients were divided into 2 groups: high expression group and low expression group. The results showed that the protein expression of Claudin-18 was closely related to nerve invasion, but not to other clinicopathological features. The incidence of nerve invasion in patients with low expression of Claudin-18 was significantly higher than that in patients with high expression of Claudin-18 $\left(x^{2}=4.321, \mathrm{P}=0.038\right)$ (Table 2).

\section{Risk factors associated with the prognosis of patients with gastric cancer}

As of June 2019, a total of 37 gastric cancer patients were followed up, and 26 cases were lost for changing the phone number. Finally, 37 participants were included in the statistical analysis. The time of operation of the 37 patients was from December 2012 to June 2013. Up to June 2019, the number of deaths was 18 (48.6\%). The survival time of the patients was 1-77 months. The Cox regression model included 37 patients to analyze the risk factors associated with the clinical prognosis of gastric cancer patients. Univariate Cox regression analysis showed that nerve invasion, Claudin-18 staining score, tumor size, and positive count of lymph nodes were risk factors associated with the prognosis of gastric cancer (Table 3).

Then, the 4 positive factors were included in multivariate
Cox regression analysis, and the results showed that Claudin-18 staining score and tumor size were independent risk factors associated with the prognosis of gastric cancer (Table 4).

Furthermore, log-rank analysis showed that the overall survival (OS) of patients with a low Claudin-18 staining score was significantly reduced in comparison to those with high staining score (log-rank $=7.225, \mathrm{P}=0.007$, Figure $2 A$ ). And the OS of patients with larger tumor size was significantly reduced compared to those with smaller tumor size $(\log$-rank $=10.579, \mathrm{P}=0.001$, Figure $2 B)$.

\section{Discussion}

The Claudin-18 protein participates in the formation of the gastric mucosal epithelial barrier and is closely related to the tissue differentiation, invasion, metastasis, and prognosis of gastric cancer. Previous studies have shown that the expression of Claudins is often reduced in cancer tissues, resulting in abnormal tight junction of tumor cells, cell differentiation, and polarity reduction (10). Our study showed that the expression of Claudin-18 was reduced in gastric cancer tissues, which was consistent with the previous report(10).

In addition, our study revealed that the incidence of nerve invasion in patients with low expression of Claudin-18 was significantly higher than that in patients with raised expression of Claudin-18, indicating that Claudin-18 expression was closely associated nerve invasion in patients with gastric cancer. Therefore, we speculated that the loss or reduction of Claudin-18 led to the destruction of tight junction integrity and the diffusion of some nutrients, and made it easier for tumor cells to leave the primary site and 
Table 2 Correlation between Claudin-18 expression and clinicopathological features

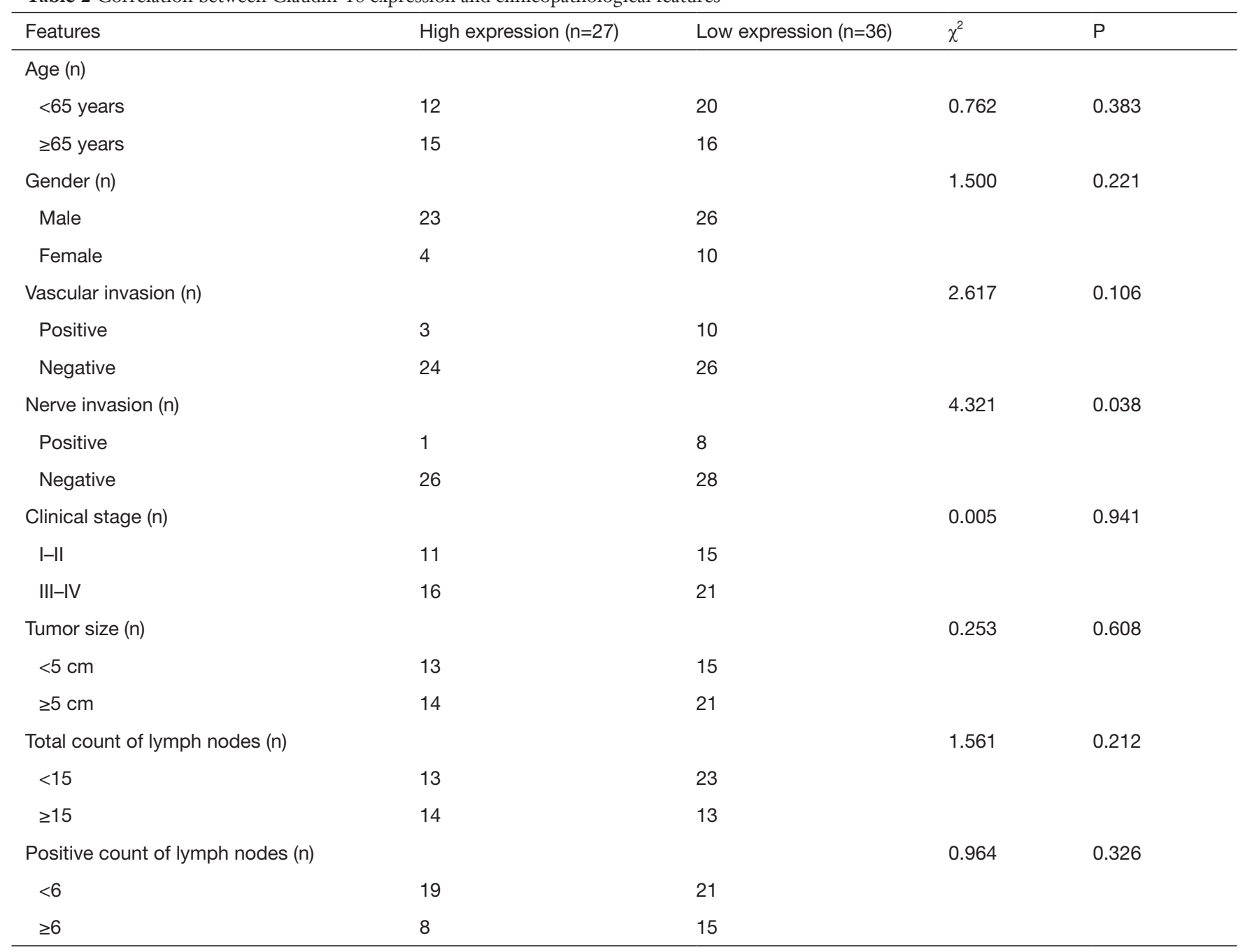

Table 3 Univariate Cox regression analysis

\begin{tabular}{|c|c|c|c|c|c|c|}
\hline Factors & B & SE & Wald & df & Sig. & $\operatorname{Exp}(B)$ \\
\hline Age & 0.024 & 0.022 & 1.154 & 1 & 0.283 & 1.024 \\
\hline Nerve invasion & -1.637 & 0.598 & 7.487 & 1 & 0.006 & 0.195 \\
\hline Clinical stage & & & 5.088 & 2 & 0.078 & \\
\hline II & -0.868 & 0.640 & 1.839 & 1 & 0.175 & 0.420 \\
\hline Claudin-18 staining score & -0.600 & 0.211 & 8.075 & 1 & 0.004 & 1.549 \\
\hline Tumor size & 0.390 & 0.099 & 15.616 & 1 & 0.000 & 1.477 \\
\hline Total count of lymph nodes & -0.010 & 0.025 & 0.170 & 1 & 0.680 & 0.990 \\
\hline
\end{tabular}

B, coefficient value; SE, standard error; df, degree of freedom; Sig, significance; Exp(B), hazard ratios. 
Table 4 Multivariate Cox regression analysis

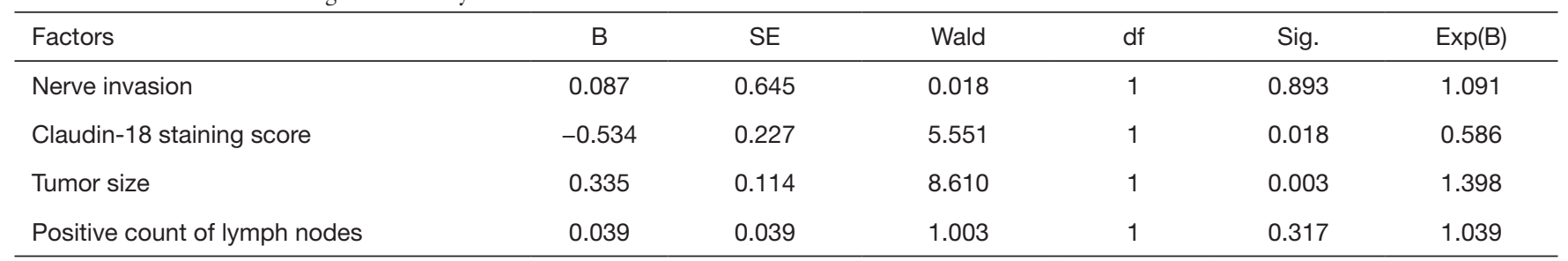

B, coefficient value; SE, standard error; df, degree of freedom; Sig, significance; Exp(B), hazard ratios.
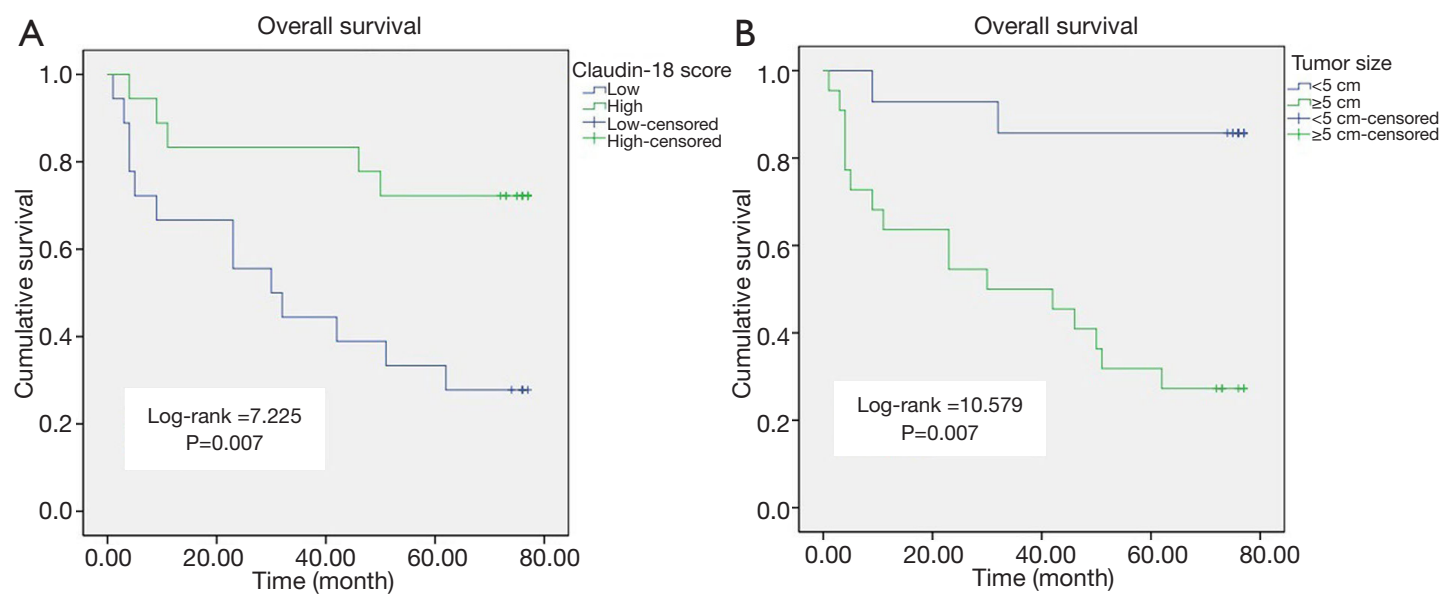

Figure 2 Log-rank analysis. (A) Claudin-18 staining score, (B) tumor size.

reach the surrounding and distant tissues, thus promoting the development of tumors $(10,11)$. To our knowledge, our study was the first to report that Claudin-18 expression was closely associated with nerve invasion in gastric cancer.

Furthermore, we analyzed the risk factors associated with the prognosis of gastric cancer. It was found that nerve invasion, Claudin-18 staining score, tumor size, and positive count of lymph nodes were risk factors associated with the survival of gastric cancer patients. Among which, the Claudin-18 staining score and tumor size were independent risk factors associated with the survival of gastric cancer patients. And log-rank analysis showed that the OS of patients with low Claudin-18 staining scores or larger tumor sizes was significantly reduced. Previous studies have shown that the survival time of patients with positive Claudin-18 expression is longer than that of patients with negative expression $(12,13)$. The results of our study were similar to previous findings, indicating that the loss or reduced expression of Claudin-18 predicted a poor prognosis for patients with gastric cancer.

Our results suggested that Claudin-18 plays an important role in the development of gastric cancer, however, the development of gastric cancer is a complex process of multistep, stage, and gene collaborative changes. The invasion, metastasis, and development of gastric cancer all involve the changes of many related genes. These genes interact, promote or inhibit each other, or regulate each other, with great complexity. Therefore, even if the loss of or reduced Claudin-18 expression is speculated to be involved in the development of gastric cancer, its role and underlying mechanism remain unresolved $(14,15)$.

There are some strengths and limitations in our study. The clinical and pathological data of gastric cancer patients collected in this study were relatively complete, which increases the reliability of the study to a certain extent. However, there are also the following shortcomings: firstly, this study included patients with preoperative metastases, and the heterogeneity of these patients may have affected the conclusion. We tried to carry out hierarchical analysis to exclude patients with metastases, but the number of cases with complete follow-up data was less than that of intake data, which may have caused statistical bias of this study to some extent; secondly, IHC was semi-quantitative. Although the double-blind scoring method was used in this 
study, it is susceptible to subjective influence and cannot eliminate measurement bias, which requires improvement for future research.

To conclude, the low expression of Claudin-18 was closely related to nerve invasion in gastric cancer, which indicated the poor clinical prognosis of gastric cancer patients. Claudin-18 is a promising prognosis predictor. The current study provides theoretical and experimental basis for further exploring the mechanism of Claudin-18 and finding new therapeutic targets.

\section{Acknowledgments}

Funding: This research was supported by the Top Talents Project of "Six-one Project" for High-level Health Talents in Jiangsu Province (LGY2018016); Key Talents Project of "Strengthening Health through Science and Education" of Wuxi Health and Family Planning Commission (ZDRC039); Application and Translation of Key Techniques on Gastrointestinal Lubricant (ZM008); the Wuxi Commission of Health (M202027); the Wuxi City Health and Family Planning Commission Youth Research Project (Q201747).

\section{Footnote}

Reporting Checklist: The authors have completed the REMARK reporting checklist. Available at http://dx.doi. org/10.21037/jgo-20-463

Data Sharing Statement: Available at http://dx.doi. org/10.21037/jgo-20-463

Conflicts of Interest: All authors have completed the ICMJE uniform disclosure form (available at http://dx.doi. org/10.21037/jgo-20-463). The authors have no conflicts of interest to declare.

Ethical Statement: The authors are accountable for all aspects of the work in ensuring that questions related to the accuracy or integrity of any part of the work are appropriately investigated and resolved. All procedures performed in this study involving human participants were in accordance with the Declaration of Helsinki (as revised in 2013). The study was approved by the ethics committee of our hospital before implementation (No. IEC20200142). The participants gave their written informed consent before participation in the study.
Open Access Statement: This is an Open Access article distributed in accordance with the Creative Commons Attribution-NonCommercial-NoDerivs 4.0 International License (CC BY-NC-ND 4.0), which permits the noncommercial replication and distribution of the article with the strict proviso that no changes or edits are made and the original work is properly cited (including links to both the formal publication through the relevant DOI and the license). See: https://creativecommons.org/licenses/by-nc-nd/4.0/.

\section{References}

1. Chen $W$, Zheng R, Baade PD, et al. Cancer statistics in China, 2015. CA Cancer J Clin 2016;66:115-32.

2. Torre LA, Bray F, Siegel RL, et al. Global cancer statistics, 2012. CA Cancer J Clin 2015;65:87-108.

3. Jindal V. Role of immune checkpoint inhibitors and novel immunotherapies in uveal melanoma. Chin Clin Oncol 2018;7:8.

4. Hargrove L, Kennedy L, Demieville J, et al. Commonly used $\mathrm{H} 1$ and $\mathrm{H} 2$ histamine receptor (HR) blockers decrease cholangiocarcinoma xenograft tumor growth, angiogenesis and EMT, Gastroenterology 2017;152:1182-3.

5. Lu R, Chen Q, Liu X, et al. Detection of circulating stage III-IV gastric cancer tumor cells based on isolation by size of epithelial tumor: using the circulating tumor cell biopsy technology. Transl Cancer Res 2019;8:1342-50.

6. Van CE, Sagaert X, Topal B, et al. Gastric cancer. J Natl Cancer 2016;57:2654-64.

7. Carpenter G, Red Brewer M. EpCAM: another surfaceto-nucleus missile. Cancer Cell 2009;15:165-6.

8. Günzel D, Yu AS. Claudins and the modulation of tight junction permeability. Physiol Rev 2013;93:525-69.

9. Lal-Nag M, Morin PJ. The claudins. Genome Biol 2009;10:235.

10. Swisshelm K, Macek R, Kubbies M. Role of claudins in tumorigenesis. Adv Drug Deliv Rev 2005;57:919-28.

11. Liu GY, Huang Z, Wang ZW. Introduction and Interpretation of TNM Staging System for Gastric Cancer in the 8th Edition of the International Anti-Cancer Alliance and the Joint American Cancer Commission. Abdominal Surgery 2017;30:241-5.

12. Jun KH, Kim JH, Jung JH, et al. Expression of claudin-7 and loss of claudin-18 correlate with poor prognosis in gastric cancer. Int J Surg 2014;12:156-62.

13. Chen SZ, Mu JC. The relationship between lymph node metastasis rate and pathological and clinical prognosis 
of gastric cancer in the elderly. Chin J Geriatrics 2015;34:1088-90.

14. Zhang WQ, Fang GL, Ma QS. Expression and clinical significance of epithelial cell adhesion molecule in gastric

Cite this article as: $\mathrm{Lu} \mathrm{Y,} \mathrm{Wu} \mathrm{T,} \mathrm{Sheng} \mathrm{Y,} \mathrm{Dai} \mathrm{Y,} \mathrm{Xia} \mathrm{B,}$ Xue Y. Correlation between Claudin-18 expression and clinicopathological features and prognosis in patients with gastric cancer. J Gastrointest Oncol 2020;11(6):1253-1260. doi: 10.21037/jgo-20-463 cancer. Guangdong Med Sci 2011;32:2815-7.

15. Figueiredo C, Garcia-Gonzalez MA, Machado JC. Molecular pathogenesis of gastric cancer. Helicobacter 2013;18 Suppl 1:28-33. 